symmetries. Moreover the original functions need not be rational; for instance in Theorem 1 the $m_{j}(>0)$ and $n_{j}(>0)$ need not be rational.

\title{
REFERENCES
}

1. M. Fekete and J. von Neumann, Über die Lage der Nullstellen gewisser Minimumpolynome, Jber. Deutsch. Math.-Verein. 31 (1922), 125-138.

2. T. S. Motzkin and J. L. Walsh, On the derivative of a polynomial and Chebyshev approximation, Proc. Amer. Math. Soc. 4 (1953), 76-87.

3. - The least pth power polynomials on a finite point set, Trans. Amer. Math. Soc. 83 (1956), 371-396.

4. J. L. Walsh, The location of critical points, Amer. Math. Soc. Colloq. Publ. Vol. 34, Amer. Math. Soc., Providence, R. I., 1950.

5. J. L. Walsh and O. Shisha, The zeros of infrapolynomials with prescribed values at given points, Proc. Amer. Math. Soc. 14 (1963), 839-844.

6. J. L. Walsh, Geometry of the zeros of the sums of linear fractions, Trans. Amer. Math. Soc. (to appear).

HARVARD UNIVERSITY AND

AERospace Research Laboratories,

Wright-Patterson Air Force Base

\section{NONLINEAR DIFFERENTIAL EQUATIONS WITH FORCING TERMS ${ }^{1}$}

\section{FRED BRAUER}

1. In this paper, we shall study the solutions of a differential equation containing a linear term with constant coefficients, a nonlinear term, and a forcing term depending only on the independent variable. We shall attempt to compare these with solutions of the equation obtained by neglecting the nonlinear term. This is a problem which frequently arises in physical examples, where the linear equation is solved and its solution is used to describe approximately the motion governed by the nonlinear equation. We shall see that the solutions of the two equations do behave similarly if the nonlinear term is small enough. This does not settle the question by any means, as in practice the nonlinear term is frequently not small enough for our result to be applicable.

Received by the editors June 14, 1963.

1 This research was supported in part by the National Science Foundation (G-24355). 
2. We wish to compare the solutions of the linear nonhomogeneous system with constant coefficients

$$
x^{\prime}=A x+p(t)
$$

with the solutions of the nonlinear system

$$
y^{\prime}=A y+f(t, y)+p(t) \text {. }
$$

Here $x$ and $y$ are $n$-dimensional column vectors, $A$ is an $n \times n$ matrix, $p$ is an $n$-dimensional column vector function continuous on $0 \leqq t<\infty$, and $f$ is an $n$-dimensional column vector function continuous on $0 \leqq t<\infty,|y|<\infty$. We will use $|y|$ to denote any suitable norm for vectors.

The comparison of (1) and (2) requires some knowledge of the linear homogeneous system

$$
x^{\prime}=A x,
$$

which has a fundamental matrix $X(t)=e^{t A}$. It is possible to make a linear change of dependent variable to bring $A$ into any desired canonical form, and it is no restriction to assume that this has been done.

It follows easily from the variation of constants formula [2, p. 74] that if all solutions of (1) are bounded on $0 \leqq t<\infty$, then all solutions of (3) are bounded on $0 \leqq t<\infty$, and this is equivalent to

$$
|X(t)| \leqq K, \quad\left|X(t) X^{-1}(s)\right| \leqq K, \quad 0 \leqq s \leqq t<\infty,
$$

for some constant $K>0$. It is easy to deduce from the variation of constants formula that the condition (4) together with the convergence of $\int_{0}^{\infty}|p(t)| d t$ implies the boundedness of all solutions of (1). However, the convergence of this integral is by no means necessary for the boundedness of solutions of (1).

We will require a bound on the nonlinear term $f$. We will assume that there exists a continuous non-negative function $\lambda$ such that

(5) $|f(t, y)| \leqq \lambda(t)|y|, 0 \leqq t<\infty,|y|<\infty$, with $\int_{0}^{\infty} \lambda(t) d t<\infty$.

This bound appears rather artificial, but we shall see in $\$ 3$ that the results of this section are not true with a weaker condition.

We require one preliminary result, part of which is essentially contained in [3].

Lemma 1. Let $\sigma$ be a positive constant and let $\lambda$ be a continuous nonnegative function on $0 \leqq t<\infty$ such that either $\int_{0}^{\infty} \lambda(s) d s<\infty$ or $\lim _{t \rightarrow \infty} \lambda(t)$ $=0$. Then $\lim _{t \rightarrow \infty} e^{-\sigma t} \int_{0}^{t} e^{\sigma \delta} \lambda(s) d s=0$. 
Proof. First we consider the case that $\lambda$ is integrable. Given any $\epsilon>0$, choose $t$ so large that $\int_{t / 2}^{\infty} \lambda(s) d s<\epsilon / 2$ and $e^{-\sigma t / 2} \int_{0}^{t / 2} \lambda(s) d s<\epsilon / 2$. Then

$$
e^{-\sigma t} \int_{0}^{t / 2} e^{\sigma s} \lambda(s) d s \leqq e^{-\sigma t / 2} \int_{0}^{t / 2} \lambda(s) d s<\epsilon / 2
$$

and

$$
e^{-\sigma t} \int_{t / 2}^{t} e^{\sigma s} \lambda(s) d s \leqq \int_{t / 2}^{t} \lambda(s) d s \leqq \int_{t / 2}^{\infty} \lambda(s) d s<\epsilon / 2 .
$$

Thus $e^{-\sigma t} \int_{0}^{t} e^{\sigma s} \lambda(s) d s / \epsilon$ for sufficiently large $t$, and $\lim _{t \rightarrow \infty} e^{-\sigma t} \int_{0}^{t} e^{\sigma s} \lambda(s) d s$ $=0$. Now consider the case that $\lambda(t) \rightarrow 0$ as $t \rightarrow \infty$. If $\int_{0}^{\infty} e^{\sigma s} \lambda(s) d s<\infty$, then the result is obvious. Otherwise, we can apply L'Hospital's rule to give

$$
\lim _{t \rightarrow \infty} \int_{0}^{t} e^{\sigma s} \lambda(s) d s / e^{\sigma t}=\lim _{t \rightarrow \infty} \lambda(t) / \sigma=0 .
$$

The systems (1) and (2) are said to be asymptotically equivalent if there is a correspondence between solutions $x(t)$ of (1) and $y(t)$ of (2) such that

$$
\lim _{t \rightarrow \infty}[y(t)-x(t)]=0
$$

THEOREM 1. If all solutions of (1) are bounded on $0 \leqq t<\infty$, and if $f$ satisfies (5), then (1) and (2) are asymptotically equivalent.

Proof. Let $\hat{x}(t)$ and $y(t)$ be solutions of (1) and (2), respectively, with $\hat{x}(0)=y(0)=y_{0}$. Then, by the variation of constants formula,

$$
\begin{aligned}
& \hat{x}(t)=X(t) y_{0}+\int_{0}^{t} X(t) X^{-1}(s) p(s) d s \\
& y(t)=X(t) y_{0}+\int_{0}^{t} X(t) X^{-1}(s) p(s) d s+\int_{0}^{t} X(t) X^{-1}(s) f(s, y(s)) d s .
\end{aligned}
$$

Thus $y(t)-\hat{x}(t)=\int_{0}^{t} X(t) X^{-1}(s) f(s, y(s)) d s, \quad$ and $\quad|y(t)-\hat{x}(t)|$ $\leqq K \int_{0}^{t} \lambda(s)|y(s)| d s$, using (4) and (5). This implies

$$
|y(t)| \leqq|\hat{x}(t)|+K \int_{0}^{t} \lambda(s)|y(s)| d s,
$$

and if $|\hat{x}(t)| \leqq \hat{L}$, the Gronwall inequality [1, p. 35] gives $|y(t)|$ $\leqq \hat{L} \exp \left[K \int_{0}^{t} \lambda(s) d s\right] \leqq \hat{L} \exp \left[K \int_{0}^{\infty} \lambda(s) d s\right]$. Since $\lambda$ is integrable, this shows that every solution of $(2)$ is bounded on $0 \leqq t<\infty$. 
Now we write $X(t)$ in the form

$$
X(t)=\left(\begin{array}{cc}
X_{1}(t) & 0 \\
0 & X_{2}(t)
\end{array}\right),
$$

where $X_{1}(t)$ and $X_{2}(t)$ are square blocks. We can also decompose the identity matrix $I$ into blocks of the same size, $I=I_{1}+I_{2}$, where

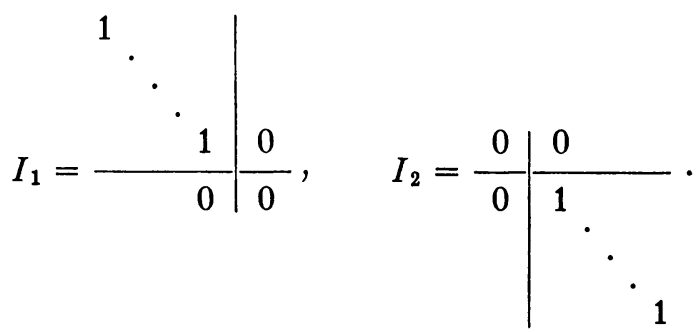

Then $X(t) X^{-1}(s)=X(t) I_{1} X^{-1}(s)+X(t) I_{2} X^{-1}(s)$. We decompose $X(t)$ in such a way that $X_{1}(t)$ contains all terms corresponding to characteristic roots of $A$ with negative real parts and $X_{2}(t)$ contains all terms corresponding to characteristic roots of $A$ with zero real part. This can be accomplished by putting $A$ into a form consisting of such blocks. Note that since all solutions of (3) are bounded, every characteristic root of $A$ with zero real part is simple. Then there exist constants $\sigma>0$ and $M \geqq 0$ such that

$$
\begin{array}{rlrl}
\left|X(t) I_{1} X^{-1}(s)\right| & \leqq M e^{-\sigma(t-s)}, & & 0 \leqq s \leqq t<\infty, \\
\left|I_{2} X^{-1}(s)\right| \leqq M, & & 0 \leqq s<\infty .
\end{array}
$$

Now we can find a constant vector $c$ such that

$$
\begin{aligned}
y(t)= & X(t) c+\int_{0}^{t} X(t) X^{-1}(s) p(s) d s+\int_{0}^{t} X(t) I_{1} X^{-1}(s) f(s, y(s)) d s \\
& -\int_{t}^{\infty} X(t) I_{2} X^{-1}(s) f(s, y(s)) d s .
\end{aligned}
$$

This follows immediately from the variation of constants formula with the choice

$$
c=y_{0}+\int_{0}^{\infty} I_{2} X^{-1}(s) f(s, y(s)) d s .
$$

The integral here is bounded by $M \int_{0}^{\infty} \lambda(s)|y(s)| d s$ (using (13)). Since $|y(s)|$ is bounded and $\lambda$ is integrable, the integral converges. Thus the representation ( 7$)$ is valid. 
We compare $y(t)$, as given by (7) with the solution $x(t)$ of (1) given by

$$
x(t)=X(t) c+\int_{0}^{t} X(t) X^{-1}(s) p(s) d s
$$

Then

$y(t)-x(t)=\int_{0}^{t} X(t) I_{1} X^{-1}(s) f(s, y(s)) d s-\int_{t}^{\infty} X(t) I_{2} X^{-1}(s) f(s, y(s)) d s$,

and, because of (6), we have

(8) $|y(t)-x(t)| \leqq M \int_{0}^{t} e^{-\sigma(t-8)} \lambda(s)|y(s)| d s+M \int_{t}^{\infty} \lambda(s)|y(s)| d s$.

Since $|y(s)|$ is bounded on $0 \leqq t<\infty$, the first integral on the right side of (8) tends to zero as $t \rightarrow \infty$ by Lemma 1 , and the second integral tends to zero because of the integrability of $\lambda$. Thus $\lim _{t \rightarrow \infty}[y(t)-x(t)]=0$, and the theorem is proved.

Of course, Theorem 1 includes the homogeneous case, $p(t) \equiv 0$, which is a result of Levinson [3]. The requirement that $A$ be a constant matrix was used only to permit the representation (7) and the estimate (6). If the constant matrix $A$ is replaced by a continuous matrix $A(t)$ and if it is known that the fundamental matrix $X(t)$ of $x^{\prime}=A(t) x$ satisfies

$$
|X(t)| \leqq K e^{-\sigma t}, \quad\left|X(t) X^{-1}(s)\right| \leqq K e^{-\sigma(t-s)}, \quad t \geqq s \geqq 0,
$$

then the proof of Theorem 1 can be carried out without decomposing $X(t)$, replacing $(7)$ by

$$
y(t)=X(t) y_{0}+\int_{0}^{t} X(t) X^{-1}(s) f(s, y(s)) d s .
$$

An estimate of the form (9) may be obtained in some cases if $A(t)$ is periodic or a constant matrix multiplied by a scalar function.

Theorem 1 can be applied to show that corresponding to every solution of the nonlinear system (2), there is a solution of the linear system (1) which behaves like it for large $t$. Thus, if there is resonance in the linear case, then there must also be resonance in the nonlinear case. However, it may be difficult to determine which solution of the linear system behaves like a given solution of the nonlinear system.

To conclude this section, we give an elementary condition which implies that all solutions of (1) tend to zero. In view of Theorem 1 , this yields a corresponding condition which implies that all solutions of (2) tend to zero. 
TheOREM 2. If $X(t)$ satisfies (9) and either $\int_{0}^{\infty}|p(t)| d t<\infty$ or $\lim _{t \rightarrow \infty} p(t)=0$, then all solutions of (1) tend to zero as $t \rightarrow \infty$. If, in addition, $f$ satisfies (5), then all solutions of (2) tend to zero as $t \rightarrow \infty$.

Proof. The variation of constants formula, together with (9), gives

$$
|x(t)| \leqq K|x(0)| e^{-\sigma t}+K \int_{0}^{t} e^{-\sigma(t-s)}|p(s)| d s
$$

for every solution $x(t)$ of (1). Lemma 1 shows that $x(t) \rightarrow 0$. The statement about solutions of (2) follows from this and Theorem 1.

3. Theorem 1 is not entirely satisfactory, because in practice it is of interest to consider a perturbation $f$ which is only assumed to be $o(|y|)$ for small $|y|$, rather than to satisfy (5). The following example shows that under this hypothesis, not only do we not have asymptotic equivalence of (1) and (2), but there may be unbounded solutions of (2) even though all solutions of (1) are bounded.

Let $x(t)$ be the solution of $x^{\prime}=b(t), x(0)=x_{0}$, where $b$ is a nonnegative integrable function and $x_{0}>0$. Then $x(t)=x_{0}+\int_{0}^{t} b(s) d s$ is a monotone increasing bounded function and tends to the limit $x_{0}+\int_{0}^{\infty} b(s) d s>0$ as $t \rightarrow \infty$. Let $y(t)$ be the solution of $y^{\prime}=b(t)+y^{2}$, $y(0)=x_{0}$. Then

$$
y(t)=x_{0}+\int_{0}^{t} b(s) d s+\int_{0}^{t}[y(s)]^{2} d s=x(t)+\int_{0}^{t}[y(s)]^{2} d s .
$$

Thus $y(t) \geqq x(t)$ for all $t$. Since $\lim _{t \rightarrow \infty} x(t)>0$, $\lim _{\inf _{t \rightarrow \infty}} y(t)>0$, and $y(t)$ cannot belong to $L^{2}(0, \infty)$. Thus $y(t)=x(t)+\int_{0}^{t}[y(s)]^{2} d s \rightarrow \infty$ as $t \rightarrow \infty$.

We can obtain some information when the perturbation $f$ is $o(|y|)$, provided the forcing function $p$ is small enough. Our result requires the use of the following form of the Gronwall inequality, more general than the form given in $[1$, p. 35$]$, but contained in the generalization in $[2$, p. 37].

Lemma 2. Suppose $r$ is a continuous non-negative function satisfying an inequality

$$
r(t) \leqq c+\int_{0}^{t}[K r(s)+\lambda(s)] d s, \quad t \geqq 0,
$$

where $c$ and $K$ are non-negative constants and $\lambda$ is a non-negative integrable function. Then 


$$
r(t) \leqq c e^{K t}+\int_{0}^{t} \lambda(s) e^{K(t-s)} d s, \quad t \geqq 0 .
$$

TheOREM 3. Suppose that $X(t)$ satisfies (9), and that for every $\epsilon>0$ there exists $\delta>0$ such that

$$
|f(t, y)| \leqq \epsilon|y|, \quad|y|<\delta .
$$

Choose $\epsilon<\sigma / K$ and suppose

$$
|p(t)| \leqq \delta(\sigma-K \epsilon) / 2 K, \quad 0 \leqq t<\infty .
$$

Then every solution $y(t)$ of (2) with $|y(0)|<\delta / 2 K$ can be continued to $0 \leqq t<\infty$, and $|y(t)|<\delta / 2$ on $0 \leqq t<\infty$.

Proof. As before, we can write

$$
y(t)=X(t) y_{0}+\int_{0}^{t} X(t) X^{-1}(s) f(s, y(s)) d s+\int_{0}^{t} X(t) X^{-1}(s) p(s) d s,
$$

and (9) and (10) give

$$
\begin{aligned}
|y(t)| \leqq & K\left|y_{0}\right| e^{-\sigma t}+\int_{0}^{t} K e^{-\sigma(t-s)} \epsilon|y(s)| d s \\
& +\int_{0}^{t} K e^{-\sigma(t-s)}|p(s)| d s,
\end{aligned}
$$

as long as $|y(t)|<\delta$. We define $r(t)=e^{\sigma t}|y(t)|$, so that

$$
r(t) \leqq K\left|y_{0}\right|+\int_{0}^{t}\left[K \epsilon r(s)+K e^{\sigma s}|p(s)|\right] d s .
$$

By Lemma 2, this implies

$$
r(t) \leqq e^{K \epsilon t}\left[K\left|y_{0}\right|+\int_{0}^{t} K e^{\sigma s}|p(s)| e^{-K \epsilon s} d s\right],
$$

or

$$
|y(t)| \leqq K\left|y_{0}\right| e^{-(\sigma-K \epsilon) t}+K e^{-(\sigma-K \epsilon) t} \int_{0}^{t}|p(s)| e^{(\sigma-K \epsilon) 8} d s .
$$

Since $\left|y_{0}\right|<\delta / 2 K$, the first term on the right side of (12) is less than $\delta / 2$ for all $t \geqq 0$.

In view of (11), the second term is no greater than

$$
\delta e^{-(\sigma-K \epsilon) t} \int_{0}^{t} e^{(\sigma-K \epsilon) s} d s / 2(\sigma-K \epsilon) \leqq \delta / 2
$$


Thus $|y(t)|<\delta$ and (12) is valid for all $t \geqq 0$. In fact, (12) gives

$$
\begin{aligned}
|y(t)| & \leqq K\left|y_{0}\right| e^{-(\sigma-K \epsilon) t}+\delta\left[1-e^{-(\sigma-K \epsilon) t}\right] / 2 \\
& \leqq \frac{\delta}{2} e^{-(\sigma-K \epsilon) t}+\frac{\delta}{2}\left[1-e^{-(\sigma-K \epsilon) t}\right]=\frac{\delta}{2} \quad \text { for } t \geqq 0 .
\end{aligned}
$$

Corollary. If, in Theorem $3, p(t) \rightarrow 0$ as $t \rightarrow \infty$, or $\int_{0}^{\infty}|p(t)| d t<\infty$, then $y(t) \rightarrow 0$ as $t \rightarrow \infty$.

Proof. We can use Lemma 1 to show that the second term on the right side of (12) tends to zero.

It is clear that the result of Theorem 3 remains true if $p(t)$ is replaced by a function $g(t, y)$, provided the bound (11) assumed for $p$ is replaced by a bound for $g$ which is uniform in $y$ for sufficiently small $|y|$. It is also clear that if the bound on $p$ is assumed only for $t \geqq T$, rather than for $t \geqq 0$, then the result remains true for a solution $y(t)$ with $|y(T)|$ sufficiently small. In this form, Theorem 1 is identical with a well-known result $[2$, p. 327$]$. However, the proof given here is more direct and gives an explicit estimate (12) of the size of the solution.

If we are given that $p(t) \rightarrow 0$ as $t \rightarrow \infty$, then we can choose $T$ sufficiently large that (11) holds for $t \geqq T$, and we can conclude that if $y(t)$ is a solution with $|y(T)|$ sufficiently small, then $y(t) \rightarrow 0$ as $t \rightarrow \infty$. However, we cannot conclude anything about the behaviour of solutions $y(t)$ with $|y(0)|$ small.

\section{BIBLIOGRAPHY}

1. L. Cesari, Asymptotic behavior and stability problems in ordinary differential equations, Springer, Berlin, 1959.

2. E. A. Coddington and N. Levinson, Theory of ordinary differential equations, McGraw-Hill, New York, 1955.

3. N. Levinson, The asymptotic behavior of a system of linear differential equations, Amer. J. Math. 68 (1946), 1-6.

UNIVERSITY OF WISCONSIN 